\title{
A PRÁTICA DA ATIVIDADE FíSICA: ESTUDO COMPARATIVO ENTRE OS ALUNOS DE GRADUAÇÃO DA UNICAMP (UNIVERSIDADE ESTADUAL DE CAMPINAS, BRASIL)
}

\author{
THE PRACTICE OF PHYSICAL ACTIVITY: A COMPARATIVE STUDY \\ AMONG UNICAMP [STATE UNIVERSITY OF CAMPINAS, BRAZIL] \\ GRADUATING STUDENTS
}

\begin{abstract}
AUTORES
Mariângela Gagliardi caro Salve ${ }^{1}$

${ }^{1}$ Departamento de Ciências do Esporte da Faculdade de Educação Física da Universidade Estadual de Campinas
\end{abstract}

\section{A PRÁTICA DA ATIVIDADE FíSICA: ESTUDO COMPARATIVO ENTRE OS ALUNOS DE GRADUAÇÃO DA UNICAMP (UNIVERSIDADE ESTADUAL DE CAMPINAS, BRASIL] 4(3): 41-47}

\section{PALAVRAS-CHAVE}

atividade física; sedentarismo; cursos de graduação.

\section{KEYWORDS}

physical activity; sedentarism; graduation courses.

data de submissão Julho 2005

data de aceitação Agosto 2007
RESUMO

A prática regular e sistematizada é fundamental para a saúde do ser humano. Diante dessa perspectiva, interessou-nos conhecer e comparar o nível de importância e os interesses dados à prática da atividade física pelos alunos da Universidade Estadual de Campinas, UNICAMP. Foram selecionados 1350 indivíduos, de ambos os sexos, com idade entre 17 e 22 anos, alunos da UNICAMP, matriculados em período integral, de nove cursos de graduação, sendo estes: Enfermagem, Educação Física, Biologia, Pedagogia, Dança, Música, História, Matemática e Computação. Pode-se observar que somente os alunos do curso de Educação Física, mais da metade (89\%), praticam atividade física de forma satisfatória com o mínimo de três vezes por semana e duração de trinta minutos. Observa-se que a prática da atividade física deve aos seguintes aspectos considerados: o combate e controle aos fatores estressantes seguido da manutenção do condicionamento físico, estética, socialização, saúde, qualidade de vida e por últimos estão - lazer e o ganho ou a manutenção da hipertrofia muscular. 0 futebol, a musculação, o condicionamento físico, corrida e a ginástica localizada foram as atividades mais procuradas. Assim, sugerimos aos coordenadores dos cursos de Graduação da UNICAMP que reservem espaços e tempo aos alunos para a prática da atividade física.

\section{ABSTRACT}

Regular and systematic practice is fundamental to the health of the human being. Facing this perspective, we became interested in knowing and comparing the level of importance and interest given to the practice of physical activity by the State University of Campinas (UNICAMP) students. 1350 Unicamp students from 17 to 22 years old, of both sexes, and enrolled in nine full-time graduation courses such as Nursery, Physical Education, Biology, Pedagogy, Dancing, Music, History, Mathematics and Computer Science, were selected. It could be observed that only in the Physical Education course, more than half of them [89\%) practiced a physical activity in a satisfactory way, that being a minimum of $\mathbf{3 0}$ minutes, three times a week. It could also be observed that the practice of a physical activity is due to the following considered aspects: Combat and control of stressing factors, followed by the maintenance of physical conditioning, esthetics, socializing, health, quality of life, and last, leisure and the gain or maintenance muscular hypertrophy. Soccer, weight lifting, physical conditioning, running and localized gymnastics were the most enrolled activities. Therefore we suggest to the coordinators of UNICAMP's graduation courses that they save time and space for students to practice a physical activity. 


\section{INTRODUC̣ÃO}

Atualmente, o movimento humano é valorizado na nossa cultura como um elemento indispensável na prevenção de desequilíbrios orgânicos, bem como um meio de se atingir o bem estar, ao combater os efeitos dos agentes estressores da vida moderna e sua repercussões no organismo humano ${ }^{17}$.

São vários os estudos que indicam os benefícios, tanto nos aspectos psicológicos como biológicos da atividade física, ou seja, melhoria da capacidade cardio-respiratória, diminuição de sintomas vasomotores, da pressão arterial, da incidência de doenças cardiovasculares, retardamento e prevenção do aparecimento de diabete melittus, da osteoporose, da obesidade, da hipertensão, redução da ocorrência de certos tipos de câncer, aumento da expectativa de vida, entre outros. No aspecto psicológico ocorre meIhoria da auto-estima, autoconceito, auto-imagem e diminuição da ansiedade e depressão $0^{10,13,22,6,11,18}$.

Dados epistemológicos mostram que a inatividade física é provavelmente um fator de risco individual para a hiperlipidemia, hipertensão, obesidade e o diabetes. As pessoas inativas, em geral, são mais hipertensas, hiperlipidêmicas, com a maior porcentagens de gorduras e propensão de tornarem-se diabéticas não dependentes de insulina. Ao passo que, as pessoas com um estilo de vida mais ativo tem maiores possibilidades de prevenir essas patologias".

De acordo com Litvak et al. ${ }^{19}$ os hábitos sociais, fumo, ingestão de álcool e de cafeína associados ao estresse e a inatividade física, contribuem para a instalação de cardiovasculares, doenças pulmonares obstrutivas crônicas, neoplasias, diabetes mellitus e cirrose. Essas doenças vêm ocupando um maior espaço de morbimortalidade de populações latino-americanas. $\mathbf{O}$ indivíduo sedentário aumenta isoladamente o risco relativo de morte em torno de $25 \%{ }^{35}$.

Conforme vários estudos mostram a prática regular e sistematizada da atividade física é fundamental na prevenção e controle das doenças crônico-degenerativas. Porém, ainda é grande a porcentagem de população inativa, ou seja, sedentária ${ }^{30}$. A maior parte da população adulta possa ser classificada como inativa, enquanto 15 a $25 \%$ seria considerada fisicamente ativa de forma regular. Existem claras evidências sob o sedentarismo como sendo o principal fator de risco coronariano, já que é relativamente alto e sua prevalência é bastante superior do que a dos outros fatores (idade, sexo, história familiar). Com a inatividade física o corpo responderá atrofiando a massa muscular, perdendo a resistência, diminuindo a massa óssea, as funções pulmonares, a flexibilidade e outras capacidades físicas ${ }^{8}$.

Constata-se, ainda, que $67 \%$ da população adulta brasileira não realiza nenhum tipo de atividade física ${ }^{3}$. Pesquisas mostram que nos Estados Unidos e na Inglaterra a taxa de inatividade física são de $24 \%$ e 15\% respectivamente. Epidemiologistas americanos, segundo estes autores, estimam que há aproximadamente 250.000 mortes por ano que poderiam ser atribuídas a inatividade física e 32.400 vidas poderiam ser salvas se houvessem incremento na atividade física. 0 Projeto Saúde (um estudo realizado pelo Ministério da Saúde sob estilo de vida) revelou que $96 \%$ das pessoas não tem um estilo de vida inteiramente saudável somente $10 \%$ da população entre 18 e 55 anos declaram fazer atividade física regularmente pelo menos três vezes por semana 20. Estudos realizados por Matsudo et $\mathrm{al}^{20}$, no CELAFISC, em escolares de São Caetano do Sul e llha Bela revelaram que os hábitos das atividades físicas das crianças brasileiras são menores e gastam mais tempo assistindo TV que as crianças americanas.

Conforme as informações do Departamento de Ergometria e Reabilitação Cardiovascular, da Sociedade Brasileira de Cardiologia, apenas de $10 \%$ dos adultos brasileiros fazem exercícios regulares mais de três vezes por semana ${ }^{15 .}$ Pode-se constatar que a prática de atividade física, segundo vários estudos, tende a ser diminuída com o passar dos anos, ou seja, o individuo quando criança pratica mais atividade física do que quando adolescente e diminui-se mais ainda sua prática quando adulto e assim por diante ${ }^{27}$. Este estilo de vida, o sedentarismo, tem sido adotado em todas as faixas etárias. Pode-se notar um aumento do número de adolescentes sedentários e indivíduos obesos, os quais adotam, com muita freqüência, o hábito de assistir televisão, existindo uma relação positiva entre o nível de adiposidade e o número de horas diárias de TV assistidas ${ }^{20}$. 0 problema agrava-se mais quando há o consumo de alimentos enquanto assistem televisã ${ }^{5}$.

Calfas et $\mathrm{al}^{7}$, descreveram que a participação de atividade física na escola é mais freqüente entre meninas $20 \%$ e nos meninos $16 \%$, considerando em três ou mais dias por semana de atividade física vigorosa. Acrescentam que entre os universitários, com idades entre 18 e 21 anos, essa prática declina para $6 \%$ tanto para homens e $7 \%$ tanto nas mulheres. $\mathbf{O}$ fato pode 
ser melhor entendido pela diminuição do tempo livre para a prática de atividade física e também pela falta de oportunidades nas aulas de Educação Física, pois na maioria das vezes os estudos e os trabalhos remunerados, entre outros, os afastam destas aulas. Quando ingressam na universidade, os jovens tem redução da acessibilidade à programas de atividade física pela diminuição de tempo. Esta transição é caracterizada pelo aumento simultâneo da demanda causada pelos estudos, semelhante ao início de carreira ou formação de nova família. Portanto, os autores concluíram que estas e outras transições da vida são períodos de riscos para a adoção de estilo de vida sedentário.

Assim, par das evidências de que o homem contemporâneo utiliza-se cada vez menos de suas potencialidades corporais e de que o baixo nível de atividade física é fator decisivo no desenvolvimento de doenças degenerativas sustenta-se a hipótese da necessidade de se promover mudanças no seu estilo de vida, levando-o a incorporar a prática de atividades físicas ao seu cotidiano ${ }^{3}$.

Nessa perspectiva, interessou-nos conhecer e comparar o nível de importância e os interesses dados à prática da atividade física pelos alunos da Universidade Estadual de Campinas, UNICAMP, que estão matriculados em período integral, a partir de duplo interesse: por um lado, trata-se de gerar elementos que permitam aprofundar o conhecimento sobre o segmento da população estudada e por outro, oportuniza-se a acumulação de subsídios, para melhor programação pedagógica da intervenção que se disponibiliza.

\begin{tabular}{llllll}
\hline & \multicolumn{2}{c}{ Homens } & & \multicolumn{2}{c}{ Mulheres } \\
\cline { 2 - 3 } \cline { 5 - 6 } & F. A. & F. R. & & F. A. & F. R. \\
\hline Sexo & 672 & 49,78 & & 678 & 50,22 \\
Idade: 17 e 19,5 anos & 224 & 33,33 & & 253 & 37,31 \\
Idade: 19,6 e 22 anos & 448 & 66,67 & & 425 & 62,69
\end{tabular}

TABELA1

Distribuição de frequência da amostra da pesquisa segundo sexo e faixas etárias.

ABREVIATURAS

F.A.: Freqüencia absoluta; F.R.: Freqüencia relativa.

\section{METODOLOGIA}

Foram respondidos 1433 protocolos, sendo selecionados 1350 . Os sujeitos da pesquisa foram alunos da UNICAMP, de ambos os sexos, com idade entre 17 e 22 anos, matriculados em período integral, de nove cursos de graduação, sendo estes: Enfermagem, Educação Física, Biologia, Pedagogia, Dança, Música, História, Matemática e Computação (tabela 1).

As pessoas foram informadas sobre os objetivos da pesquisa e o caráter anônimo da mesma, bem como tinham a liberdade de participação e de desistência, colhendo-se a seguir, o termo de assentimento esclarecido. A pesquisa foi aprovada pelo Comitê de Ética da FCM/UNICAMP (Parecer 371/2001). A esses indivíduos foi garantida a não divulgação de seus nomes.

Não expressaram inadequações nas respostas aos quesitos do instrumento adotado, sendo considerado válido e confiável.

\section{Procedimentos}

Consideraram-se como apropriado numa perspectiva quantitativa/ /descritiva, este estudo é observacional descritivo seccional ou de corte transversal, o qual, segundo Pereira $^{6}$ e Almeida Filho ${ }^{2}$, consiste de investigação que produz instantâneos da situação de saúde de grupo ou comunidade, evidenciando as características apresentadas naquele momento através de amostras representativas da população. 0 protocolo de investigação consistiu de sete questões sobre o tema investigado. A primeira parte continha questões a informar a idade, sexo e profissão, em seguida vieram as relacionadas com a atividade física. Esse foi respondido pelos próprios sujeitos.

$\mathrm{O}$ instrumento de coleta de dados foi elaborado a partir de adaptação de outros disponíveis em pesquisas anteriores de Ramos $^{27}$ e Samulski e Noce ${ }^{34}$.

\section{Estatística}

A análise estatística foi executada através do pacote SPSS for Windows, onde foram processadas as informações pertinentes, após respectivo armazenamento dos dados coletados. São apresentadas séries de distribuições de freqüências absolutas e relativas, sob forma tabular, de acordo com Padovani (2001).

\section{RESULTADOS}

A partir da coleta dos materiais foram apresentados os seguintes resultados (ver tabelas 2,3 e 4). 


\begin{tabular}{lccccc}
\hline & \multicolumn{2}{c}{ Praticantes } & & \multicolumn{2}{c}{ Não Praticantes } \\
\cline { 2 - 3 } \cline { 5 - 6 } Cursos de graduação & F. A. & F. R. & & F. A. & F. R. \\
\cline { 2 - 3 } Enfermagem & 48 & 72 & & 102 & 28 \\
Educação Física & 89 & 134 & & 61 & 16 \\
Biologia & 38 & 57 & & 112 & 43 \\
Dança & 31 & 46,5 & & 120 & 53,5 \\
Pedagogia & 32 & 48 & & 118 & 52 \\
Música & 21 & 31,50 & & 129 & 68,50 \\
História & 18 & 27 & & 132 & 73 \\
Matemática & 42 & 63 & & 108 & 37 \\
Computação & 31 & 46,50 & & 119 & 53,50 \\
TotAL & & & & & 100 \\
\hline
\end{tabular}

\section{TABELA2}

Distribuição de freqüência da prática da atividade física, com o mínimo da três vezes por semana e duração de trinta minutos, segundo nove cursos de graduação da UNICAMP.

ABREVIATURAS

F.A.: Freqüencia absoluta; F.R.: Freqüencia relativa.

\section{DISCUSSÃO}

Dos que praticam regularmente atividade física foram apontados as seguintes motivações (ver tabela 3). A tabela 4 mostra as modalidades esportivas mais praticadas pelos alunos. vezes por semana e duração de trinta minutos, são indivíduos mais ativos fisicamente. Em seguida aparecem os cursos de Enfermagem [48\%], Matemática (42\%), Biologia (38\%), Pedagogia (32\%), Computação [31\%], Dança [30\%), Música (21\%] e por último lugar História [18\%].

Das pessoas sedentárias ou dos que não praticam atividade física de forma satisfatória foram apontados os principais motivos para a não adoção de um estilo de vida ativa: falta de tempo, extensa carga horária de estudo e a falta de prazer. Observa-se, tabela 3 , que a prática da atividade física deve-se, aos aspectos considerados importantes, o combate e controle aos fatores estressantes seguido da manutenção do condicionamento físico, estética, socialização, saúde, qualidade de vida e por últimos estão o lazer e o ganho ou a manutenção da hipertrofia muscular.

Segundo Matsudo et al. ${ }^{20}$ a respeito da aderência ao exercício físico, relatam que esta resulta entre outros fatores, da existência de variáveis positivas, a saber: instrução adequada, rotina regular, ausência de lesões, diversão, alegria e variedade, grupo camarada, conhecimento do progresso, aprovação do parceiro.

Em uma pesquisa efetuada por Chagas e Samuski apud Samuski ${ }^{33}$ os autores também encontraram a estética, o condicionamento físico, a melhora do estado de saúde e por último prazer, as razões das práticas de atividades físicas.

De forma semelhante, Okuma ${ }^{23}$ a melhora da aparência e o reduzir o estresse, são os principais fatores que fazem com que determinado indivíduo freqüente os programas de exercícios físicos regulares.

Distribuição de frequêencia da motivação da prática da atividade física, segundo nove cursos de graduação da UNICAMP.

ABREVIATURAS

F.A.: Freqüencia absoluta; F.R.: Freqüencia relativa. 
Diferente do nosso resultado encontrado, onde o controle do estresse foi apontado como o principal motivo da prática da atividade física, nas pesquisas de Ramos et al. ${ }^{28} \mathrm{e}$ de Pohl et al. ${ }^{25}$ encontraram-se que aspecto saúde foi considerado elemento motivacional.

Já, ao contrario das pesquisas anteriores, Carvalho et al. ${ }^{8}$ detectaram que o motivo saúde não foi aspecto prioritário, aparecendo em quinto lugar, com 8,50\%. Essa pesquisa teve o intuito de detectar os objetivos que levam os indivíduos a ingressarem em academia de ginástica em Divinópolis-MG. A análise dos dados foi obtida através de verificação dos objetivos relatados pelos próprios alunos durante a anamnese presente na avaliação. $A$ amostra constitui-se de $\mathbf{8 8 0}$ alunos.

Analogamente ao resultado da nossa pesquisa, Dantas ${ }^{12}$ identificou que os interesses que impulsionam a procura de atividade física em academia, procurando identificar o perfil social a partir das ligações que se estabelecem entre as motivações e as atividades físicas escolhidas. Foram avaliadas $\mathbf{3 0 0}$ pessoas que freqüentam academia em Maceió e para tal utilizou-se um questionário. Concluiu-se também, a saúde não é uma razão prioritária pela procura da prática desportiva, aparecendo em terceiro lugar com $13,8 \%$.

0 condicionamento físico foi outro elemento detectado, em nossa pesquisa, pela busca da prática desportiva situando-se em segundo lugar, da mesma forma que nos estudos de Carvalho et al. ${ }^{8}$ e Ramos et al. ${ }^{27}$. Quanto aos outros itens apontados como relevantes foram: estética, socialização, ganho ou manutenção da hipertrofia muscular e a qualidade de vida.

\begin{tabular}{lcc} 
Modalidades desportivas & F. A. & F. R. \\
\hline $\begin{array}{l}\text { Futebol de campo } \\
\text { Musculação, Condicionamento Físico }\end{array}$ & 3 & 33,33 \\
$\begin{array}{l}\text { Corrida Ginástica localizada, } \\
\text { Corrida de longa duração }\end{array}$ & 2 & 22,22 \\
$\begin{array}{l}\text { Caminhada, Futsal, Basquetebol, } \\
\text { Voleibol, Natação }\end{array}$ & 1 & 11,11 \\
\hline
\end{tabular}

TABELA4

Freqüência das modalidades desportivas praticadas, segundo os nove cursos de graduação da UNICAMP.

ABREVIATURAS

F.A.: Freqüencia absoluta; F.R.: Freqüencia relativa.

Na pesquisa de Rufino et al. ${ }^{29}$ a estética foi também valorizada. Os autores tiveram o propósito de verificar algumas características de freqüentadores de academias de ginástica no estado do Rio Grande do Sul. Utilizou-se como instrumento de coleta de dados questionário contendo 12 questões fechadas relativas a idade, sexo, profissão, nível de instrução, renda mensal, atividade física praticada, tempo, freqüência, objetivos, satisfação, nível de informação e fonte de informação. A amostra foi composta por todos os freqüentadores de academias de ginástica do estado que se dispusessem a participar. Analisaram 1139 questionários, através dos quais obteve-se, entre outros resultados, que a maior parte foi composta por mulheres com idade entre 16 e 25 anos. Tais resultados vão ao encontro dos de $\mathrm{Saba}^{30}$ e o de Dantas $^{12}$ que citam que o que levam os indivíduos as academia é a meIhoria da estética.

Ramos $^{27}$ também constatou que os alunos universitários também buscam a estética corporal. Escreve que "as pessoas estão em constantes pressões para ter o corpo perfeito que vêem na mídia e fazem qualquer coisa para atingi-lo", geralmente, associam a rigorosas dietas alimentares.

No estudo de Moritz ${ }^{21}$ com alunos universitários da Universidade Federal do Paraná concluiu que as alunas participam mais de atividade física como experiência social, visando estética, já os alunos da mesma Universidade participam mais de atividade física em busca de emoções.

A procura dos graduandos pela atividade física como uma atividade de lazer é um aspecto importante a ser considerado. 0 mesmo foi observado pelos alunos dos cursos de extensão oferecido pela Faculdade de Educação Física da UNI$\mathrm{CAMP}^{27}$. Rufino et al. ${ }^{29}$ citam que uma das razões pelos quais as pessoas freqüentam as academias é o lazer associada à socialização.

Segundo Stah $^{32}$ para os universitários, estar junto de amigos e familiares tem influência positiva na participação de atividade física. Ramos $^{27}$ encontrou porcentagens ainda menores do que o que nos encontramos, através de questionário composto de 14 questões, entre 246 alunos da UNICAMP, sendo 137 homens e 109 mulheres, 
os quais $28,86 \%$ dos alunos que entram na universidade apresentam vida ativa fisicamente, ou seja, realizam exercícios três ou mais vezes por semana, e 32,93\% são considerados intermediários, isto é, praticam atividade física uma ou duas vezes por semana.

A tabela 4 retrata que o futebol, com $33,33 \%$, é a modalidade mais praticada pelos alunos dos cursos de Educação Física, Música e Matemática. A musculação e o condicionamento físico, com $22,22 \%$, foram indicados pelos discentes dos cursos de Biologia, Educação Física e Enfermagem. A corrida de longa duração $(22,22 \%)$ pelos cursos de Enfermagem e Educação Física. A Ginástica localizada [22,22\%] pela dança. A Caminhada $(11,11 \%)$ pela Enfermagem. 0 Futsal $(11,11 \%)$, o Basquetebol [11,11\%] pela Matemática e a Natação $(11,11 \%)$ pelo curso de Letras.

Com efeito, nota-se que, nas sociedades modernas, a prática regular e sistematizada fica restrita a um grupo de indivíduos, onde o mesmo foi observado pelos alunos de graduação da UNICAMP. Tendo em vista esta problemática e a importância da atividade física torna-se essencial o enfoque da promoção e estímulo de estilo de vida ativo.

Assim, sugerimos aos coordenadores dos cursos de graduação que reservem espaços e tempo aos alunos para a prática de atividade física, baseado nos valores do corpo esbelto, da estética, do combate ao estresse e da busca da saúde e também tendo em vista que as atividades mais procuradas: o futebol de campo, condicionamento físico, musculação e a corrida.

\section{CORRESPONDÊNCIA}

Cidade Universitária "Zeferino Vaz"

Campinas, São Paulo, Brasil

CEP: 13.083 .970

C.P.: 6134

E-mail: gagliardi@fef.unicamp.br

\section{REFERÊNCIAS}

1. Achour Jr (1996). Efeitos das atividades físicas nos componentes herdados predisponentes a doenças cardiovasulares. Rev. Bras. Ativ. Fís. Saúde 1(4):53-62.

2. Almeida Filho N; Rouquariol MZ (1990). Introdução à epidemiologia moderna. Rio de Janeiro: Abrasco.

3. Assupção Lot; Moraes PP; Fontoura H (2002). Relação entre atividade física, saúde e qualidade de vida. Rev. Digital :52.

4. Barbanti W (1991). Aptidão Física e Saúde. Rev. Festur 3(1):5-8.

5. Bar-or O; Foreyty J; Bouchard C; Brownell KD; Dietz WH; Ravussin E; Arline D; Salbe AD; Schwrnger S; ST. Jeor S; Torun B (1998). Physical activity, genetic, and nutrition considerations in chidhood weight menagement. Med. Sci. Sports Exerc. 30(10):2-10.

6. Bluenthal Ja; Emery Cf; Walsh Ma; Cox Dr; Kuhn Cm; Williams Rb (1988). Exercise training in healthy type A middle-aged men. Effects on behavioral and cardiovascular responses. Psychosomatic Med. 50:418-433.

7. Calfas Kj; Sallis Jf; Nichols Jf (2000). Project grad: two years outcomes of a randomized controlled physical activity intervention among young adults. Am. J. Prev. Méd. 18:28-36.
8. Carvalho RVG (2003). Perfil de demanda de alunos egressos em academia de ginástica. In: Anais... XXVI Simpósio Internacional de Ciências do Esporte. Atividade física Construindo Saúde São Paulo, 94.

9. Carvalho T (1995). Doenças crônico-degenerativas no Brasil In: Brasil, Ministério da Saúde-Coordenação de Doenças Crônico-Degenerativas. Orientações básicas sobre atividade física e saúde para os profissionais das áreas de educação saúde. Brasília: Ministério da Saúde: 15-26.

10. Corbin C; Lindsey R (1997). Concepts of physical fitness: Brown \& Bechmark Pubilshers.

11. Crews Dj; Landers DJ (1987). A meta-analitic review of aerobic fitness and reactivity of psychosocial stressors. Med. Sci. Sports Exerc. 19 [suppl.):114-120.

12. Dantas M do SM. Perfil social e motivações dos usuários de academias de ginástica da cidade Maceió. Dissertação (Mestrado em Educação Física) - PPGEF, Universidade Gama Filho, 1998.

13. Dishman RK (1994). Introduction: consensus, problems, and prospects. In Dishman RK ed. Advances in exercise adherence. Champaign: Human Kinetics, 1-28. 14. Dishman RK (1993). Exercise adherence. In: Singer RN \& Tennant LK, ed. Handbook of Research Sport Psychology. New York: McMillan: 779-97.

15. DERC (1999):

http://www.cardiol..br/derc/posci ent.htam. 2/05/1999, 10:10 horas.

16. Fiatarone M; Marks Ec; Ryan Nd; Merdith Cn; Lipsitz La; Evans WJ (1990). High intensity strength training in monagenarians. Journal of the American Medical Association 263:3029-3034. 
17. Fernandes Filho JA (2003). A prática da avaliação física. 2 ed. Rio de Janeiro: Shape.

18. Guthrie Jr; Smiht Ama; Dennerstein L; Morse C (1995). Physical activity and menopause experience: a cross-sectional study. Maatiritas 20:71.

19. Litvak $J$ et al. (1987). The growing noncommunacable disease burden, a challenge for the conuntries of the Americas. Bull. Pan Am Health Organ. 21:156-171.

20. Matsudo V et al. (1996) Exercício e Qualidade de Vida. In: Anais... Congresso Latino Americano. Esporte, Educação e Saúde, Foz do Iguaçu, 1996. Foz do Iguaçu:85-89.

21. Moritz MR. Atitudes de universitários da Universidade Federal do Paraná em relação a prática de atividade Física e Desportos. São Paulo. Dissertação de Mestrado, Escola de Educação Física, USP, 1988.

22. Nieman DC (1998). The exercise-health connection. Champaign: Human Kinetics.

23. Okuma SS (1994). Fatores de adesão e desistência das pessoas aos programas de atividade física. In: Anais... Semana da Educação Física. São Paulo. São Paulo: Departamento de Educação Física.
Faculdades de ciências Biológicas e da Saúde. Universidade são Judas Tadeu: 30-6.

24. Padovani CR (2001). Noções básicas de bioestatística. In: Campana AO et al., Investigação científica na área medica. São Paulo: Manole, 153-202.

25. Pohl HH (2000). Importância da ginástica laboral no resgate da corporiedade. Cinergis 1(2):77-107. 26. Pereira MG (1990). Epidemiologia: teoria e prática. Rio de Janeiro: Abrasco.

27. Ramos MG. Explorando as relacões do grau de sedentarismo de alunos ingressantes na Universidade Estadual de Campinas. Campinas. Dissertação (Mestrado em Educação Física). Faculdade de Educação Física, UNICAMP, 1998. 28. Ramos Mg Galdi Eh Salve Mgc Gonçalves A [2004). Atividade Física em extensão universitária. Estudo descritivo sobre o perfil de usuários na UNICAMP. In: Pelegrinotti IL Performance Humana: saúde e esporte. Ribeirão Preto: Tecmeed, 139-157.

29. Rufino Vs; Soares Lf Da S. Santos DL (2000). Características de freqüentadores de academias de ginástica do Rio Grande do Sul. Kinesis 22:57-68.
30. Saba FKF. Determinantes da prática de exercícios físicos em academias de ginástica. Dissertação (Mestrado em Educação física). São Paulo, Faculdade de Educação Física-Unicamp, 1999.

31. Salve MGC (2003). Análise da intervenção de um programa de atividade física nos hábitos de lazer Rev. Bras. de Saúde Ocup. 28(105/106):73-82.

32. Stahl T; Rutten A; Nutbeam D; Bauman A; Kannas L et al. (2001). The importance of the social environment for physically active lifestyle-results from INTERNATIONAL TUDY. Social Science and Medicine 52:1-10.

33. Samulski DM (2002). Psicologia do esporte. Taubaté: Manole.

34. Samulski Dm; Noce F (2000). A importância da atividade física para a saúde e qualidade de vida: um estudo comparativo entre professo-res, alunos e funcionários da UFMG. Rev. Bras. Ativ. Fís. Saúde. 5(1).

35. Waib Ph; Burini RC (1995). Efeitos do Condicionamento Físico Aeróbio no Controle da Pressão Arterial. Arq Bras Cardiol. Botucatu 64(3):243-246. 